\title{
Parathyroid hormone regulates fates of murine osteoblast precursors in vivo
}

\author{
Deepak H. Balani, ${ }^{1}$ Noriaki Ono, ${ }^{1,2}$ and Henry M. Kronenberg ${ }^{1}$ \\ ${ }^{1}$ Endocrine Unit, Massachusetts General Hospital and Harvard Medical School, Boston, Massachusetts, USA. ${ }^{2}$ Department of Orthodontics and Pediatric Dentistry, \\ University of Michigan School of Dentistry, Ann Arbor, Michigan, USA.
}

\begin{abstract}
Teriparatide, a recombinant form of parathyroid hormone (PTH), is the only approved treatment for osteoporosis that increases the rate of bone formation. Teriparatide increases osteoblast numbers by suppressing osteoblast apoptosis and activating bone-lining cells. No direct evidence for teriparatide's actions on early cells of the osteoblast lineage has been demonstrated. Here, we have employed a lineage-tracing strategy that uses a tamoxifen-dependent, promoter-driven cre to mark early cells of the osteoblast lineage in adult mice. We show that teriparatide increases the numbers of osteoblast precursors and drives their differentiation into mature osteoblasts. Unexpectedly, following withdrawal of teriparatide therapy, bone marrow adipocytes increased dramatically in number. Some of these adipocytes derived from cells marked by Sox9-cre expression weeks earlier. Continued therapy with teriparatide prevented the appearance of adipocytes. Selective, inducible deletion of the PTH receptor in Sox9-cre cells demonstrated that PTH receptor expression is required for teriparatide-mediated increases in early osteoblast precursors. The increase in early precursors after teriparatide administration was associated with robust suppression of precursor apoptosis without affecting their rate of proliferation. Thus, teriparatide increases the numbers of early cells of the osteoblast lineage, hastens their differentiation into osteoblasts, and suppresses their differentiation into adipocytes in vivo.
\end{abstract}

\section{Introduction}

Parathyroid hormone (PTH) acts as a major regulator of mammalian calcium homeostasis, partly through actions on bone (1). When PTH 1-34 is administered by once-daily subcutaneous injection to humans or rodents, bone mass is increased due to an increased number of osteoblasts and bone formation rate (2). Teriparatide is currently the only agent with bone-building properties (so-called anabolic agents) approved by the FDA for treatment of people with osteoporosis. The cause of the increased number of osteoblasts after teriparatide administration has been studied extensively. Teriparatide suppresses osteoblast apoptosis (3) and activates dormant bone-lining cells so that they become active osteoblasts (4). Both of these actions of teriparatide involve postmitotic, mature cells of the osteoblast lineage. It is less clear whether teriparatide also acts on early osteoprogenitors to increase the number of new osteoblasts, partly because the identification of such cells in vivo has been challenging.

Mammalian bone marrow contains cells capable of forming colonies in culture (CFU fibroblasts [CFU-Fs]) that contain cells capable of differentiating into osteoblasts, chondrocytes, and adipocytes in vitro or after subcutaneous transplantation. These stromal cells, sometimes called mesenchymal stem cells (MSCs), have been partially purified and have been studied extensively as potential tools for regenerating bone and other tissues (5). Their normal fates in vivo remain elusive, however, because specific marking

Conflict of interest: The authors have declared that no conflict of interest exists

Submitted: November 9, 2016; Accepted: June 8, 2017.

Reference information: J Clin Invest. 2017;127(9):3327-3338.

https://doi.org/10.1172/JC191699. of these cells in lineage-tracing experiments in vivo has proven challenging. Nestin promoter-driven GFP (nestin-GFP) was first used to mark mesenchymal precursors that could become CFU-F and osteoblasts, chondrocytes, or adipocytes in vitro (6). Lineagetracing experiments using nestin-creERT 2 mice crossed with mice expressing different cell-specific reporters suggest that precursors marked with this transgene can become osteoblasts (6), though this fate may occur very infrequently. Other groups have subsequently used a variety of transgenes to mark stromal cells and follow their fates as they became osteoblasts. These transgenes use promoters such as those for $\alpha$-smooth muscle actin, MX1 stimulated with polyI-polyC, gremlin, and leptin receptor, reviewed in Ono and Kronenberg (7). Further, to identify cells in fetal and early postnatal life capable of differentiating into chondrocytes, adipocytes, and fetal and early postnatal osteoblasts, Ono et al. (8) used collagen II-creERT2, Sox9-creERT2, and aggrecan-creERT2 to mark such cells. Each of the promoters mentioned here is capable of marking mesenchymal cells that can become osteoblasts, but the specific properties of each of these mesenchymal cells differ. These differences probably reflect the heterogeneity of mesenchymal cells capable of differentiating into osteoblasts.

The ability to identify osteoblast precursors in vivo makes it possible to determine whether the action of teriparatide to increase bone mass includes actions on these precursors. When teriparatide was administered to mice expressing nestin-GFP, the number of these cells increased (6). These cells expressed the PTH/PTHrP receptor, raising the possibility that $\mathrm{PTH}$ might act directly on these cells. Here, we have chosen to use transgenic mice expressing Sox9-creERT2; R26RTomato reporter to study the role of osteoblast precursors in participating in the increase in osteoblast num- 
bers after administration of teriparatide. We also examine the fates of such cells after cessation of teriparatide administration.

\section{Results}

Sox9-creERT2 ${ }^{+}$cells label very early cells of the osteoblast lineage in early postnatal mice in vivo. To assess the contribution of cells expressing Sox9-creERT2; R26RTomato and their descendants to the osteoblastic lineage in postnatal mice, we pulsed Sox9creERT2; R26RTomato; osteocalcin-GFPtopaz (Ocn-GFPtpz) mice with administration of $0.2 \mathrm{mg}$ tamoxifen at P3. Ocn-GFPtpz mice constitutively express the topaz variant of GFP under the control of the mouse osteocalcin promoter. We did not detect tamoxifen-independent tdTomato $^{+}$cells postnatally. Four days after a tamoxifen pulse at P3 (Sox9-creERT2 ${ }^{\mathrm{P} 3}$ ), chondrocytes in the growth plate and articular surfaces as well as cells in the metaphyseal spongiosa and adjacent to the perichondrium/periosteum and endosteum were labeled by Sox9-creERT2 (Figure 1A and Supplemental Figure 3, A-C; supplemental material available online with this article; https://doi.org/10.1172/JCI91699DS1). By 27 days after the pulse, Sox 9 -creERT2 ${ }^{\mathrm{P} 3}$ cells had become osteoblasts (as judged by the yellow appearance of cells expressing both tdTomato and GFPtpz) and stromal cells in the metaphysis, epiphysis, and diaphysis in addition to chondrocytes in the growth plate and articular chondrocytes (Figure 1B and Supplemental Figure 3, D-F). At P7, we observed no overlap between tomato and GFPtpz ${ }^{+}$ cells in any region of the limb. When mice given tamoxifen were followed until P30, we observed a 42-fold increase in the number of tdTomato ${ }^{+}$cells ( $4 \mathrm{~d}$ : $8.5 \pm 2.12$ cells vs. $27 \mathrm{~d}: 360.5 \pm 28.9$ cells) in the metaphysis (Figure 1C) (see Supplemental Figure 11). We also observed an increase in the contribution of Sox9-creERT2; $R 26 R$ Tomato $^{+}$cells into the Ocn-GFPtpz population (from no cells 4 days after tamoxifen to $108 \pm 13.43$ cells at 27 days after tamoxifen) in the metaphyseal area (Figure 1E). Cell counting was performed in a region representative of metaphysis and cortical bone that was kept constant throughout the experiments. We observed a 5 -fold increase in the number of tdTomato $^{+}$cells at the cortical surface ( 4 d: $32 \pm 7.07$ vs. 27 d: $183.5 \pm 17.68$ ) (Figure 1D). We also observed that the contribution of Sox9-creER; R26RTomato ${ }^{+}$ cells to the Ocn-GFPtpz population increased in the cortical bone (from 0 at P7 to $86 \pm 4.2$ cells at P30) (Figure $1 F$ ). Thus, shortly after administration of tamoxifen, Sox9-creERT2; R26RTomato ${ }^{+}$ cells were found in the metaphysis and near bone surfaces; 27 days later, these cells had increased in number and become stromal cells and osteoblasts (Figure 1B, arrows, Figure 1, C-F, and Supplemental Figure 3, A-F). When mice were evaluated at P60 after tamoxifen administration, we observed several Sox9-creERT2; $R 26$ RTomato $^{+}$cells were colabeled with perilipin, fatty acid-binding protein 4 (FABP4), and adiponectin in the distal metaphysis (Figure 1G, Supplemental Figure 3, G-I, and Supplemental Figure 8), suggesting that Sox9-creERT2 cells are precursors of both osteoblasts and adipocytes.

Sox9-creERT2 labels undifferentiated mesenchymal precursors in adult mice in vivo. As cells expressing Sox9-creERT2 in early postnatal life include early cells in the osteoblast lineage, we next investigated whether this transgene is also active in older mice. For this purpose, 6-week-old (P42) Sox9-creERT2; R26RTomato; Ocn-GFPtpz mice were pulsed with a single $2 \mathrm{mg}$ dose of tamoxifen
(Figure 2A). tdTomato $^{+}$cells were observed at P44 in the growthplate chondrocytes, articular chondrocytes, metaphyseal spongiosa, endosteum, periosteum, and perichondrium (Figure 2B and Supplemental Figure 4, A and B). Following immunohistochemistry with anti-Sox9 Ab, we detected growth-plate chondrocytes and a few cells within the primary spongiosa that expressed the transcription factor Sox 9 in large enough amounts to be detected with $\mathrm{Ab}$ and also expressed tdTomato (Figure 2C and Supplemental Figure 4, C-E). We also confirmed that Sox9-creERT2-TdTomato ${ }^{+}$ cells isolated from the bone expressed transcripts encoding Sox9 (Figure 2D). Furthermore, we detected no tamoxifen-independent cre recombinase activity at this stage (Supplemental Figure 4, F-I). We used collagen II-creERT; R26RTomato and Aggrecan-creERT; $R 26 R$ Tomato mice and observed a very similar distribution of tomato-positive cells. However, we chose not to use those mice further because of higher tamoxifen-independent tomato reporter activity associated with their use (Supplemental Figure 2).

Next, to determine whether, as expected, Sox9-creERT2 marks early cells in the osteoblast lineage, we investigated whether Sox9creERT2 labels cells before expression of the transcription factor osterix. Because we had access to a mouse expressing osterix promoter-driven mCherry (Osx-mCherry) and the spectrum of emission of mCherry fluorophore overlaps with that of tdTomato, we performed this experiment using an R26RZsgreen1 reporter to identify cells descended from Sox9-creERT2-expressing cells. We pulsed 6-week-old Sox9-creERT2; R26RZsgreen1; Osx-mCherry mice with $2 \mathrm{mg}$ tamoxifen. We observed that the majority of Sox9-creERT2+ cells lacked mCherry protein expression both in the metaphysis (Figure 2, E, F, and H, and Supplemental Figure 4, J-Q) and on the endocortical surfaces (Figure $2 \mathrm{G}$ ). However, after several weeks, we observed Sox9-creERT2 ${ }^{+}$cells that showed overlap of ZsGreen1 expression with mCherry protein expression (Supplemental Figure 1), suggesting that a subset of Sox9-creERT2 $2^{+}$cells represents precursors of osterix-expressing cells at early times points after tamoxifen labels cells but before the expression of the transcription factor. Next, we asked whether Sox9-creERT2 ${ }^{+}$cells marked 2 days after tamoxifen administration also express nestin-GFP or CXCL12GFP, probable markers of some early cells in the osteoblast lineage $(6,9)$ or Ocn-GFPtpz (osteoblast marker). To answer this question, we crossed Sox9-creERT2; R26RTomato with either nestin-GFP or CXCL12-GFP mice. At P44, 9.81\% $\pm 3 \%$ of the Sox9-creERT2 ${ }^{+}$cells also expressed nestin-GFP (Figure 2I) and $2.89 \% \pm 0.59 \%$ expressed CXCL12-GFP (Figure 2J), but none expressed any detectable OcnGFPtpz (Figure 2K). Thus, overall Sox9-creERT2 ${ }^{+}$cells, soon after tamoxifen administration, did not overlap with osterix-expressing cells, but showed some overlap with cells marked by other known putative markers of mesenchymal precursors.

Once-daily teriparatide administration increases the numbers of descendants of Sox9-creERT2-expressing precursors and their differentiation into osteoblasts in vivo. To determine whether teriparatide administered by once-daily subcutaneous injection affected early descendants of Sox9-creERT2-expressing precursors, we pulsed 6-week-old mice with a single $2 \mathrm{mg}$ dose of tamoxifen and subsequently sacrificed these mice after 3,7 , and 21 days of administration of teriparatide or vehicle (Figure 3A). On day 3, we observed cells expressing tdTomato in the metaphyseal spongiosa and endocortical and periosteal surfaces in both vehicle- and teripa- 

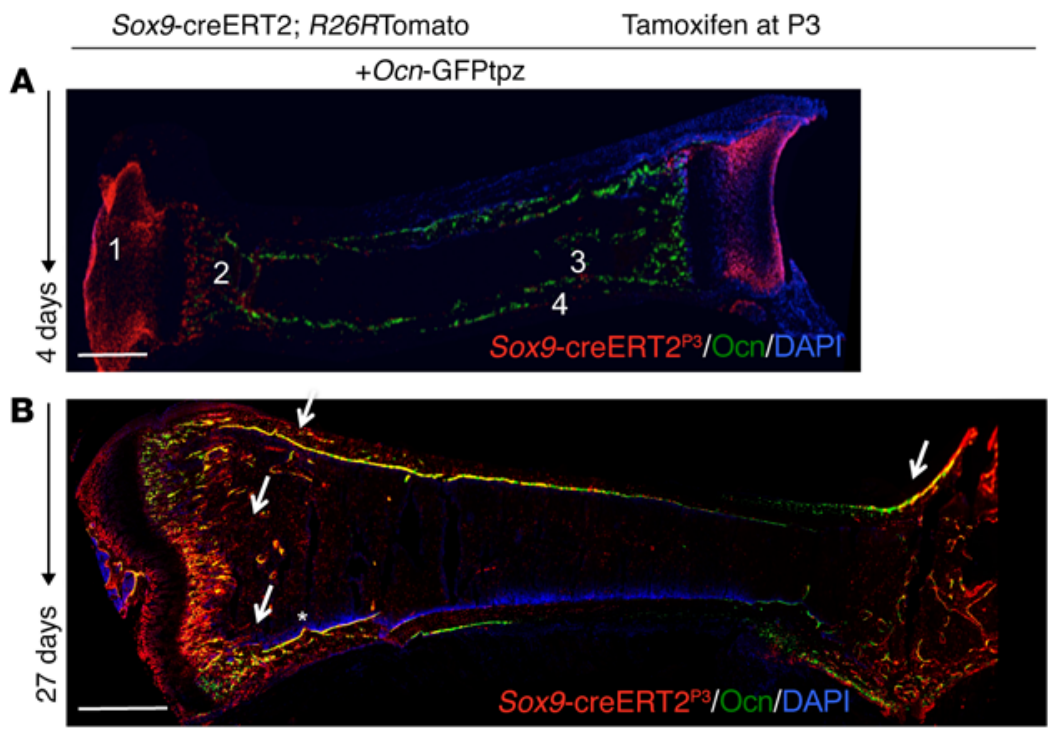

C

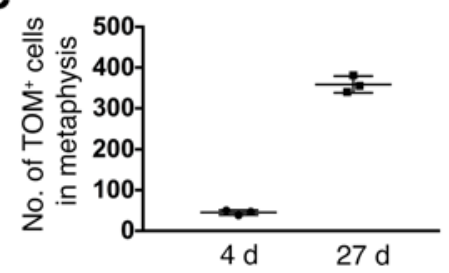

E

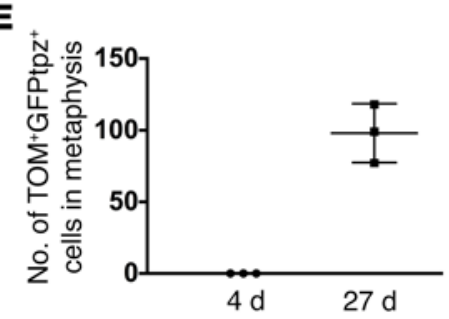

D

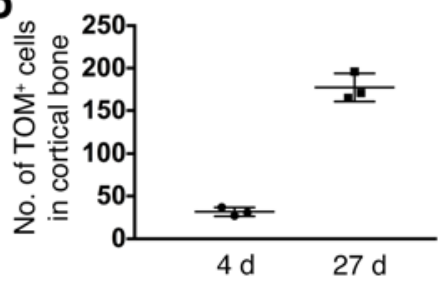

$\mathbf{F}$

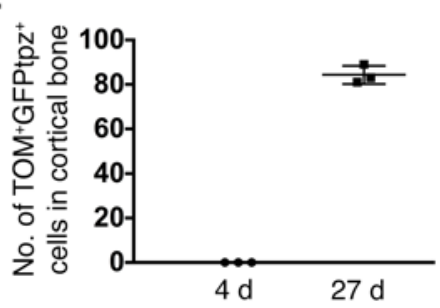

G Sox9-creERT2; R26RTomato

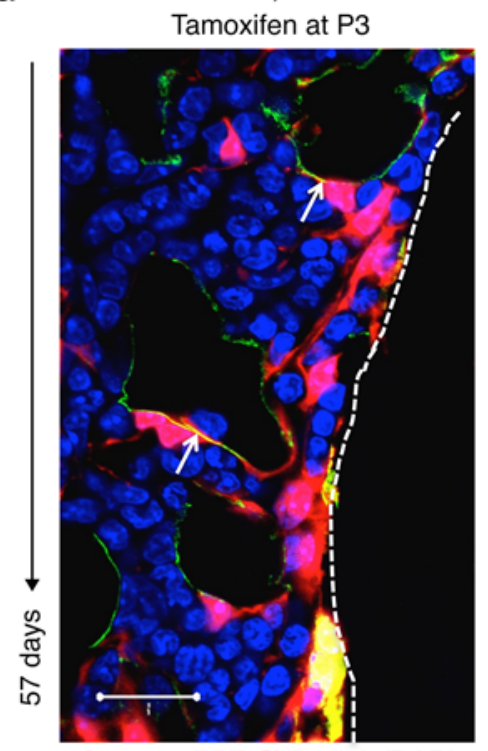

Sox9-creERT2P3/Perlipin/DAPI

Figure 1. Sox9-creERT2+ cells label early cells of the osteoblast lineage in postnatal mice in vivo. (A and B) Representative long bone section from Sox9creERT2; R26RTomato; Ocn-GFPtpz mice at 4 days (A) and 27 days (B) after tamoxifen injection. Sox9-creERT2; R26RTomato cells at P7 were seen as articular chondrocytes (no. 1), in the metaphysis (no. 2), and at endocortical (no. 3) and periosteal (no. 4) surfaces and did not overlap with Ocn-GFPtpz. At P30, several Sox9-creERT2; R26RTomato cells coincided with Ocn-GFPtpz cells in the metaphysis and at endocortical and periosteal surfaces, shown as yellow cells (arrows). Scale bars: $0.5 \mathrm{~mm}$. (C and $\mathbf{D})$ The number of Sox9-creERT2; R26RTdTomato (TOM+) cells counted in the metaphysis and cortical diaphyseal bone in standard regions described in Supplemental Figure 11 on day 4 and day 27 after tamoxifen injection. (E and F) The number of Sox 9 creERT2; R26RTomato+ (TOM+GFPtpz ${ }^{+}$) cells that also colocalized Ocn-GFPtpz protein in the metaphysis and cortical bone in diaphysis counted on day 4 and day 27 after tamoxifen injection. Data represent mean \pm SD from 3 independent experiments with 3 mice/experiment. (G) Representative confocal image of Sox9-creERT2; R26RTdTomato and perilipin-positive adipocytes (arrows) in the distal tibia after 57 days of tamoxifen administration. Data represent 3 independent experiments with 3 mice/experiment.

ratide-treated mice (Figure 3, B and E). On day 3, the distribution and numbers of tdTomato-expressing cells did not change with PTH 1-34 treatment in the metaphysis (Supplemental Figure 5, A and B) or cortical bone of the diaphysis (Supplemental Figure 5, $\mathrm{G}$ and $\mathrm{H})$. We observed $40 \pm 21$ tdTomato $^{+}$cells per section in the metaphyseal spongiosa of vehicle-treated mice compared with 42 \pm 6 cells per section in teriparatide-treated animals at day 3 (Figure $3 \mathrm{H})$. Similarly, cortical tdTomato ${ }^{+}$cells present on the endosteum and periosteum showed no significant difference in numbers (16 \pm 3 /section vs. $15 \pm 9$ /section) (Figure 3I). In addition, the fraction of tdTomato ${ }^{+}$cells that differentiated into Ocn-GFPtpz osteoblasts also showed no significant differences in mice treated with vehicle or teriparatide ( $5 \pm 2 /$ section vs. $3 \pm 1 /$ section in metaphysis and $2.5 \pm 1$ /section vs. $2 \pm 1$ /section in cortical bone) (Figure 3 , J and K) at day 3. However, by day 7, PTH 1-34 had caused striking differences in both the metaphyseal spongiosa and cortical bone of teriparatide-treated mice compared with vehicle-treated mice (Figure 3, C and F). The tdTomato ${ }^{+}$population in vehicle-treated mice remained largely undifferentiated and showed tdTomatoonly fluorescence in both metaphyseal spongiosa (Figure 3C and Supplemental Figure 5C) and cortical bone (Supplemental Figure 5I). We observed that a fraction of the tdTomato ${ }^{+}$population colocalized with GFPtpz fluorescence, indicating an increase in the differentiation of tdTomato ${ }^{+}$cells in teriparatide-treated mice 
Tamoxifen at $\mathrm{P} 42$

Sox9-creERT2; R26RTomato
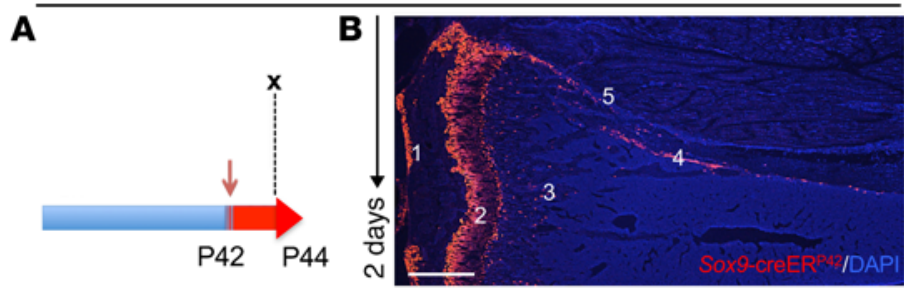

C

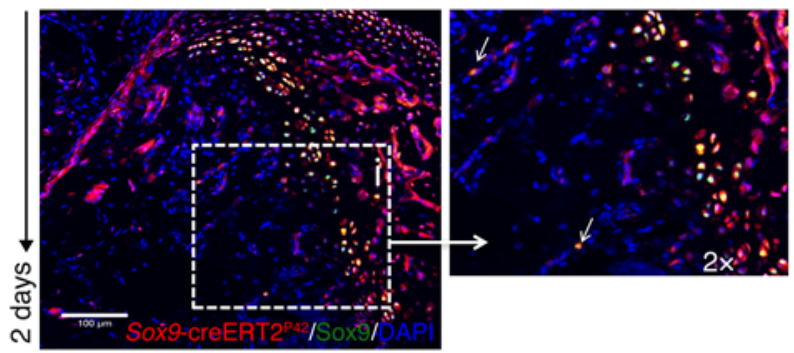

D

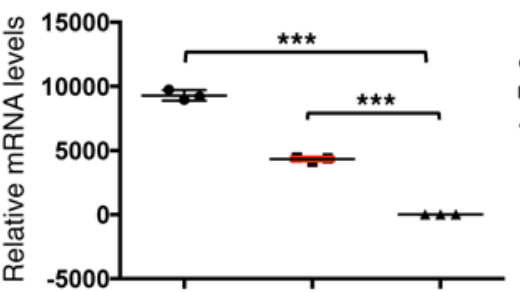

E Sox9-creERT2; R26RZsgreen1; Osx-mcherry
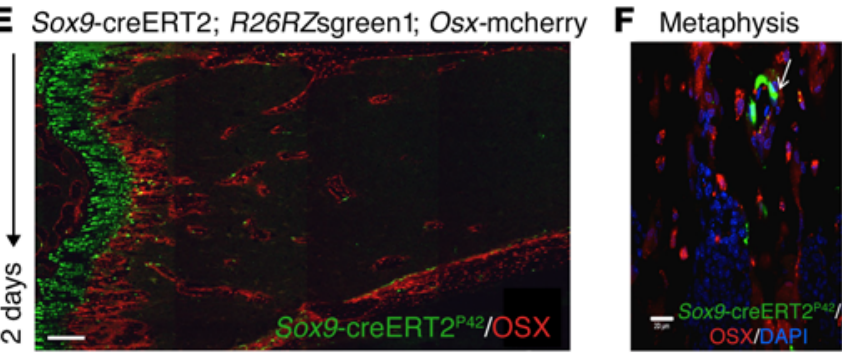

G

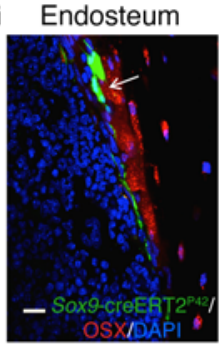

Tamoxifen at P42

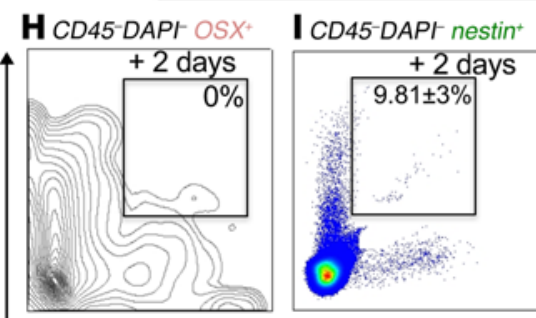

J CD45-DAPI CXCL12+ K CD45-DAPr Ocn ${ }^{+}$
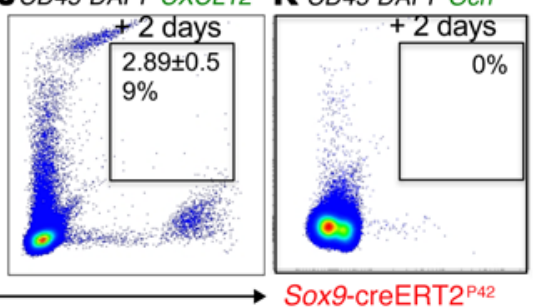

Figure 2. Sox9-creERT2+ cells are undifferentiated mesenchymal precursors in adult mice in vivo. (A) Lineage tracing of adult Sox9-creERT2; R26RTomato mice was performed by injecting $2 \mathrm{mg}$ tamoxifen (red arrow) into Sox9-creERT2; R26RTomato mice at $P 42$. Each panel reflects data from 3 mice/genotype from 3 independent experiments. (B) Representative tibia from Sox9-creERT2; R26RTomato mice 2 days after tamoxifen administration. Sox9-creERT2; $R 26 R$ Tomato cells at P44 were seen as (no. 1) articular chondrocytes, (no. 2) growth plate chondrocytes, (no. 3) metaphysis, (no. 4) endocortical, and (no. 5) on periosteal surfaces. (C) Staining with anti-Sox9 Ab overlapped with tomato expression in the growth plate and, occasionally, in the metaphysis (arrows) of Sox9-creERT2; R26RTomato mice. Right, magnified image showing overlapping of Td Tomato ${ }^{+}$and antiSox9 Ab staining (arrows). (D) Quantitative RT-PCR of Sox9 transcript levels (normalized to Gapdh). Data represent mean \pm SD from 3 independent experiments. Data were subjected to Bonferroni's correction for multiple testing. ${ }^{* *} P<0.0001$. (E) Representative section of tibia from Sox9-creERT2; R26RZsgreen1; Osx-mCherry mice. Note that most of the Osx-mCherry expression did not overlap with Zsgreen1 protein. (F and $\mathbf{G}$ ) Representative sections of higher magnification confocal images of (C) metaphysis and $(\mathbf{H})$ endocortical surface of tibia of Sox9-creERT2; R26RZsgreen1; Osx-mCherry mice, pointing to Sox9-creERT2; Zsgreen $1^{+}$cells in the metaphysis and endocortical surface that do not express osterix 2 days after tamoxifen (arrows). Sox9-creERT2; Zsgreen $1^{+}$cells ultimately gave rise to Osx-mCherry ${ }^{+}$cells when followed for 3 weeks after tamoxifen-induced labeling (Supplemental Figure 1). (H-K) Representative flow cytometry dot plot analysis showing, on day 2, lack of overlap of Osx$\mathrm{mCherry}^{+}$cells with Sox9-creERT2; R26R Tomato $^{+}$cells (H). Note that a fraction of nestin ${ }^{+}$cells overlapped with Sox9-creERT2; Zsgreen 1+ cells (I). A small fraction of $\mathrm{CXCL} 12-\mathrm{CFP}^{+}$cells also overlapped with Sox9-creERT2; R26RTomato ${ }^{+}$cells (J). No overlap was seen between Sox9-creERT2 ${ }^{+}$cells and $\mathrm{Ocn}^{-\mathrm{GFP}^{+}}$cells (K). The data represent mean \pm SD from 3 mice from 3 independent experiments. Scale bars: $500 \mu \mathrm{m}$ (B); $100 \mu \mathrm{m}$ (C (insert magnified 2x), E); $20 \mu \mathrm{m}$ (F, G). in the metaphysis (Supplemental Figure 5, C and D) and cortical bone (Supplemental Figure 5, I and J). The tdTomato ${ }^{+}$cells in metaphyseal spongiosa (119 $\pm 27 /$ section vs. $267 \pm 39 /$ section $)$ and cortical bone ( $35 \pm 6 /$ section vs. $58 \pm 3$ /section) were significantly higher in number in teriparatide-treated mice (Figure 3, H and I). Similarly, an increase in the differentiation of tdTomato $^{+}$cells into mature osteoblasts was observed (Figure 3, J and K). On day 21, teriparatide-treated mice showed an overall increase in the number of the tdTomato ${ }^{+}$population (Figure $3 \mathrm{G}$ ) in comparison with that in vehicle-treated animals (Figure 3D). A large number of metaphyseal tdTomato ${ }^{+}$cells was observed in teriparatide-treated animals ( $134 \pm 38$ /section vs. $484 \pm 73$ /section), including several that colocalized with GFPtpz protein; however, many were still present in a less differentiated state (Supplemental Figure 5E). In vehicle-treated mice, metaphyseal tdTomato ${ }^{+}$cells that colocalized GFPtpz were present ( $41 \pm 25$ /section), but these were far fewer than in comparison with those in teriparatide-treated animals (146 \pm 19$)$ (Supplemental Figure 5F and Figure 3, D, G, and J). Cortical bone appeared to have merged with trabecular bone and showed a significantly higher number of $\mathrm{tdTomato}^{+}$cells in teriparatide-treated animals ( $45 \pm 1$ /section vs. $64 \pm 2$ /section, Figure 3I and Supplemental Figure 5, K and L), though many had differentiated into osteocytes that were identified under the microscope by their typical morphology. Thus, in both vehicle-treated and terip- 


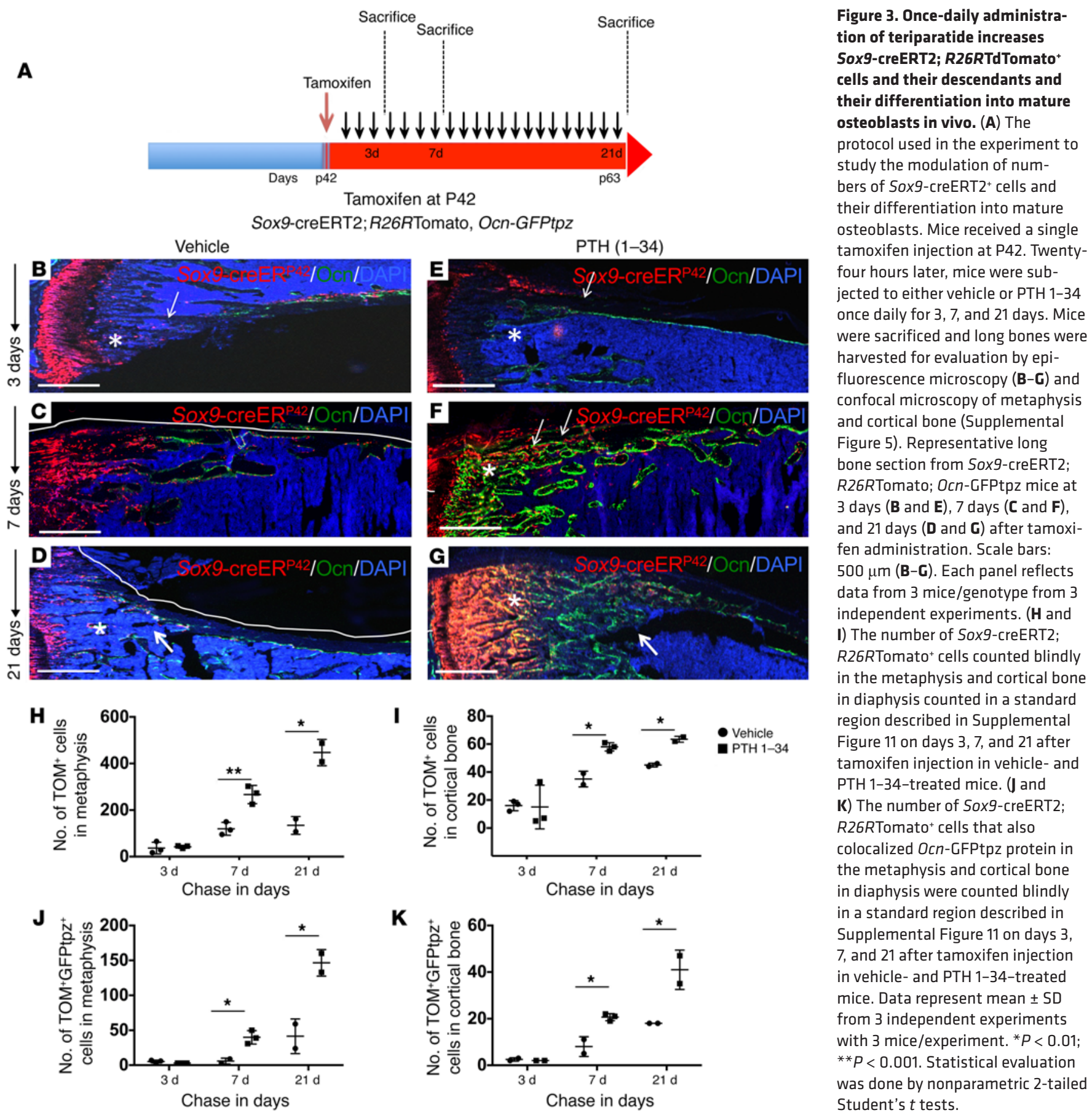

aratide-treated mice, tdTomato $^{+}$cells first appeared away from the bone surface and then increased in number and became OcnGFPtpz-expressing cells, most of which were on bone surfaces. The number of such cells and their rate of appearance were hastened by intermittent delivery of PTH 1-34. In order to determine whether teriparatide administration affects the number of very early Sox9-creERT2-positive multipotent cells, teriparatide was administered within 3, 7, or 21 days and then $2 \mathrm{mg}$ tamoxifen was administered to each group. Mice were sacrificed 24 hours after tamoxifen administration (see schematic in Supplemental Figure 6A). We observed that at all time points, teriparatide-treated mice showed a higher number of tdTomato ${ }^{+}$cells compared with vehicle in the metaphysis with the numbers at day 7 and 21 reaching statistical significance $(19 \pm 3.4,9 \pm 0$ in vehicle-treated mice vs. $141 \pm$ $33.71,75 \pm 4.1$ in teriparatide-treated mice) on day 7 and day 21 of teriparatide administration (Supplemental Figure 6B). Similarly, td Tomato ${ }^{+}$cells were higher in number in the cortical bone on day 7 in teriparatide-treated mice compared with vehicle controls $(8 \pm$ 4.35 in vehicle-treated mice vs. $25.33 \pm 8.08$ in teriparatide-treated mice), though not after day 3 and day 21 (Supplemental Figure 6C). These findings suggest that PTH 1-34 plays an important role in augmenting the number of Sox9-creERT2 $2^{+}$mesenchymal precursors and concomitantly facilitates their differentiation toward the osteoblastic lineage. 

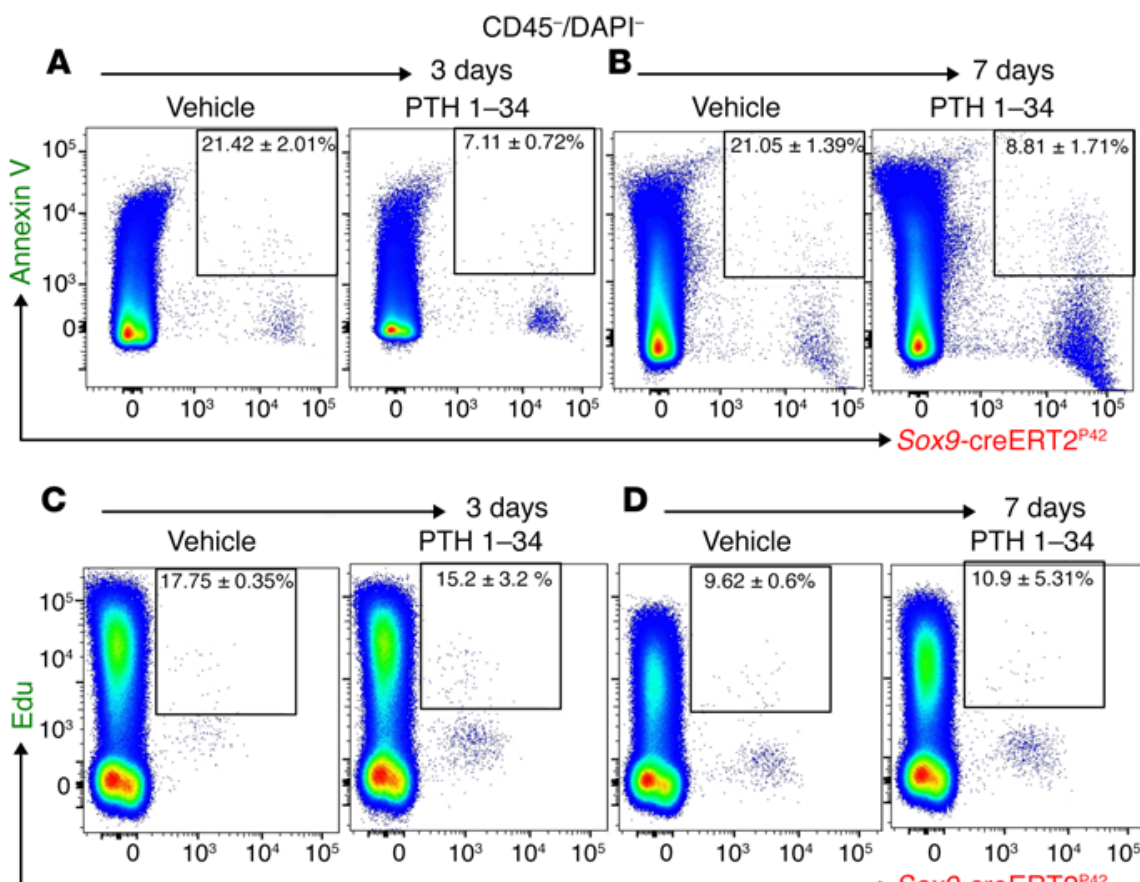

D
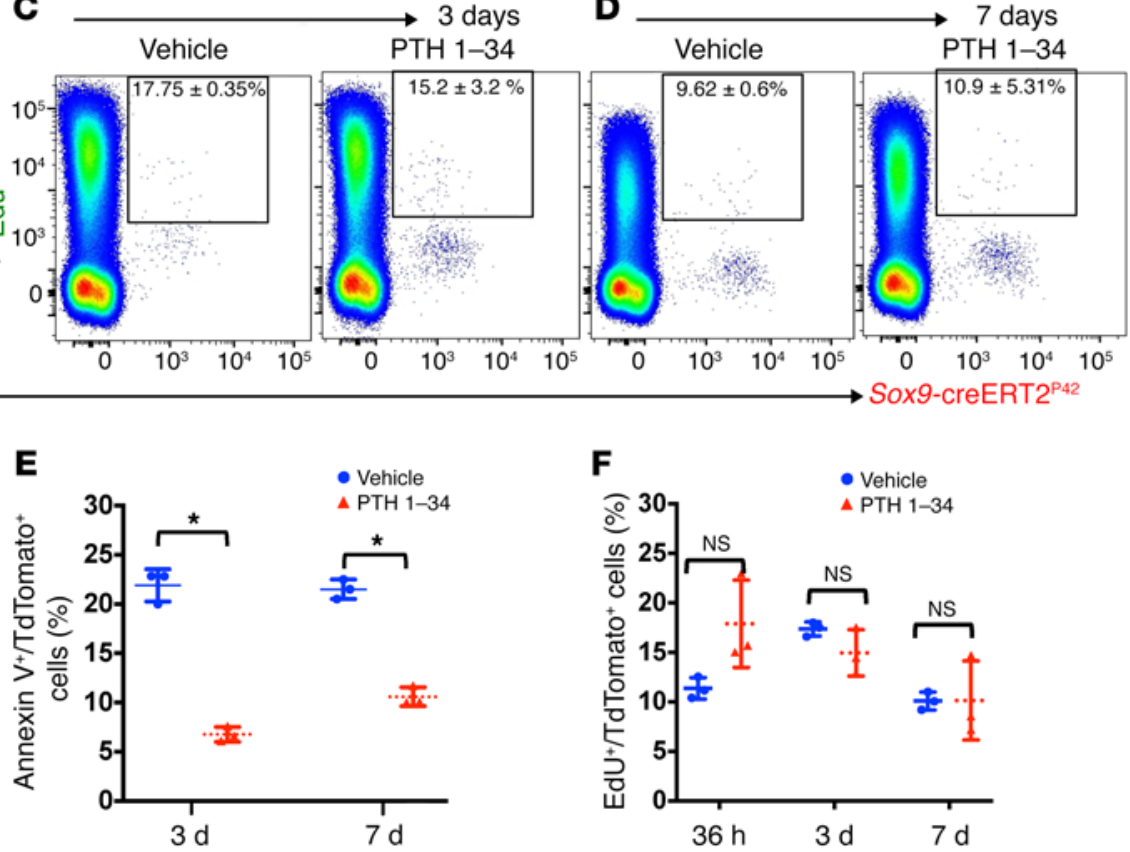

Figure 4. Teriparatide administration suppresses apoptosis in Sox9-creERT2+ multipotential cells. (A and B) Representative flow cytometry dot plot analysis showing percentage of TdTomato ${ }^{+}$cells from Sox9-creER; R26RTomato mice and gated on TdTomato ${ }^{+}$cells (after gating out all CD45 ${ }^{+}$and DAPI ${ }^{+}$cells) and annexin V-FITC at 3 and 7 days after tamoxifen. (C and D) Representative flow cytometry dot plot analysis showing percentage of TdTomato cells isolated from Sox9-creERT2; R26RTomato mice and gated on TdTomato ${ }^{+}$cells and EdU ${ }^{+}$cells at 3 and 7 days after tamoxifen. (E and $\mathbf{F}$ ) Graphs representing flow cytometry analysis of the rate of apoptosis (A and $\mathbf{B}$ ) and proliferation (C and $\mathbf{D}) \mathbf{3}$ and 7 days after tamoxifen administration. Statistical evaluation was done by nonparametric 2-tailed Student's $t$ tests. ${ }^{*} P<0.01$.
Teriparatide administration suppresses apoptosis in Sox9creERT2-positive multipotent cells. To assess the mechanism by which teriparatide administration leads to an increase in the number of Sox9-creERT2-positive multipotent cells, we performed flow cytometry on bone cells from vehicle- or teriparatide-treated mice at day 3 and day 7 after tamoxifen injection. We utilized a commercially available annexin V-FITC staining kit and labeled Sox9-creERT2; R26R Tomato $^{+}$cells. Hematopoietic cells and dead cells were gated out by anti-CD45 Ab and DAPI staining, respectively. We observed a suppression of apoptosis in the tdTomato $^{+}$population in teriparatide-treated mice compared with vehicle-treated mice on both day $3(7.11 \% \pm 0.72 \%$ in teriparatidetreated mice vs. $21.42 \% \pm 2.01 \%$ in vehicle-treated mice) and day $7(8.81 \% \pm 1.71 \%$ in teriparatide-treated mice vs. $21.5 \% \pm 1.39 \%$ in vehicle-treated mice) (Figure 4, A, B, and E). To assess whether teriparatide treatment stimulated proliferation of Sox9-creERT2 ${ }^{+}$ cells, Sox9-creERT2; R26RTomato mice received $1.5 \mathrm{mg}$ of EdU intraperitoneally 6 hours before sacrifice after treatment with vehicle or teriparatide for $1.5,3$, or 7 days. Hematopoietic cells were gated out with anti-CD45 Ab. We observed no significant differences in the rate of proliferation between vehicle- and teriparatide-treated mice (Figure 4, C, D, and F). Thus, teriparatide administration regulates the number of mesenchymal precursors by suppressing their rate of apoptosis without affecting their rate of proliferation in vivo.

Teriparatide administration increases the number of Sox9creERT2-positive cells and their differentiation into the osteoblastic lineage by direct signaling via PTH1R in vivo. To assess whether the mechanism of teriparatide-mediated increase in Sox9-creERT2positive multipotent cells and their differentiation into osteoblastic lineage is via PTH1R signaling, we created Sox9-creERT2; R26RTomato; Ocn-GFPtpz; PTH1R ${ }^{f / f l}$ transgenic mice. In PTH1R $R^{f / f l}$ mice, the Pth1r locus contains a $3^{\prime}$ loxP site and a second loxP site in intron 1. After tamoxifen administration, this model allows simultaneous labeling of cells expressing Sox9-creERT2 with tdTomato and permits deletion of the PTH1R.

Sox9-creERT2; R26RTomato; PTH1R ${ }^{f / f l}$ triple-transgenic mice and Sox9-creERT2; R26RTomato; PTH1 $R^{f / W T}$ mice received $2 \mathrm{mg}$ tamoxifen intraperitoneally and received vehicle or teriparatide administration for 7 days. We confirmed complete knockdown of transcripts encoding PTH1R in FACS-sorted tdTomato ${ }^{+}$ cells from Sox9-creERT2; R26RTomato; Ocn-GFPtpz; PTH1R ${ }^{f / f l}$ triple-transgenic mice 2 days after tamoxifen injection (Figure 5J). In Sox9-creERT2; R26RTomato; Ocn-GFPtpz; PTH1R ${ }^{f / W T}$ mice, teriparatide administration significantly $(P<0.01)$ increased the number of tdTomato ${ }^{+}$cells in metaphysis and cortical bone $(25.16$ 
Tamoxifen at P42
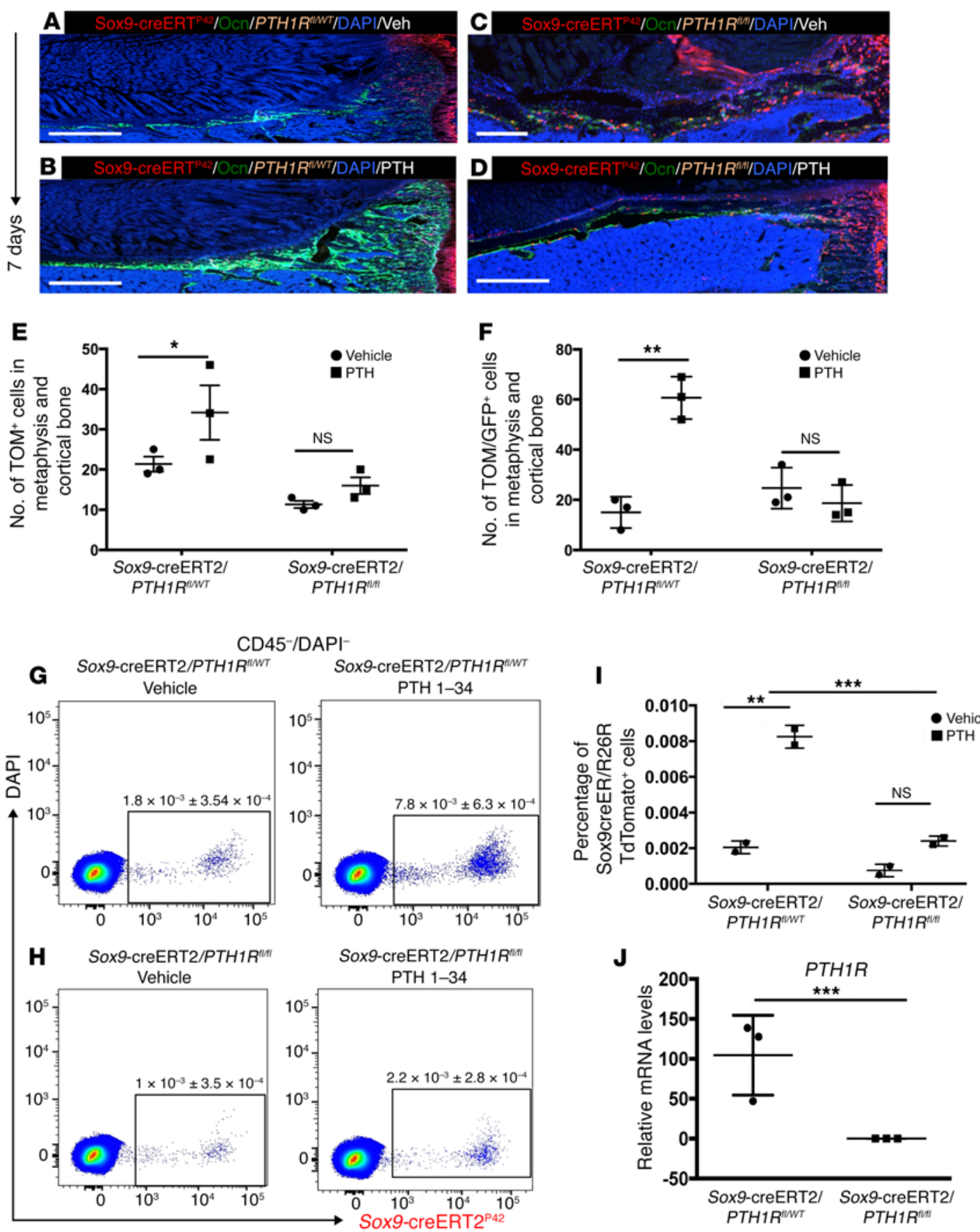

Figure 5. Teriparatide administration increases Sox9-creERT2-positive multipotent cells and their differentiation into osteoblast lineage by direct signaling via PTH1R in vivo. (A and B) Representative long bone section from vehicle- and teriparatide-treated Sox9-creERT2; R26RTomato; Ocn-GFPtpz; $P T H 1 R^{f / W T}$ mice at 7 days after tamoxifen administration. (C and $\left.\mathbf{D}\right)$ Representative long bone section from vehicle- and teriparatide-treated Sox9-creERT2; R26RTomato; Ocn-GFPtpz; PTH1R ${ }^{f l / f l}$ mice at 7 days after tamoxifen administration. Scale bars: $500 \mu \mathrm{m}$. (E) The number of Sox9-creERT2; R26RTomato cells counted blindly in the metaphysis and cortical bone in diaphysis of Sox9-creERT2; R26RTomato; Ocn-GFPtpz; PTH1R ${ }^{f / W T}$ (Sox9creER/PPR ${ }^{\mathrm{fl} / \mathrm{WT}}$ ) and Sox9-creERT2; R26RTomato; Ocn-GFPtpz; PTH1R ${ }^{f / f l}$ (Sox9creER/PPR ${ }^{f / / f 1}$ ) mice counted on day 7 after tamoxifen injection in vehicle and PTH 1-34-treated mice. (F) The number of Sox9-creERT2; R26RTomato cells counted that coincided with Ocn-GFPtpz in the metaphysis and cortical bone in diaphysis of Sox9-creERT2; R26RTomato; Ocn-GFPtpz; PTH1R ${ }^{\text {f/WT }}$ (Sox9-creER/PPR fl/WT) and Sox9-creERT2; R26RTomato; Ocn-GFPtpz: PTH1R fl/fl (Sox9-creER/PPR ${ }^{\text {fl/fl) }}$ mice counted on day 7 after tamoxifen injection in vehicle and PTH 1-34-treated mice. (G and $\mathbf{H})$ Representative flow cytometry dot plot analysis showing percentage of TdTomato ${ }^{+}$cells from Sox9-creERT2; R26RTomato mice obtained by serial digestion and 7 days after tamoxifen. (I and J) Representative bar graphs showing transcripts encoding PTH1R in sorted TdTomato+ cells 2 days after tamoxifen injection. Data represent mean \pm SD from 3 independent experiments with 3 mice/experiment. ${ }^{*} P<0.01$; ${ }^{* *} P<0.001$; ${ }^{* *} P<0.0001$. Statistical evaluation was done by nonparametric 2-tailed Student's $t$ tests and the data were subjected to Bonferroni's correction for multiple testing. 
\pm 2.75 in vehicle-treated mice vs. $53.6 \pm 5.77$ in teriparatide-treated mice). However, deletion of PTH1R led to complete abrogation of increase in the number of tdTomato $^{+}$cells in the metaphysis and cortical bone (10.6 \pm 6.42 in vehicle-treated mice vs. $7.6 \pm 2.51$ in teriparatide-treated mice) (Figure 5, A-F). Similarly, the colocalization of $\mathrm{TdTomato}^{+}$cells with Ocn-GFPtpz in metaphysis and cortical bone was observed to occur normally in the absence of $\mathrm{PTH}$, but with no increase in response to PTH in mice lacking the PTH receptor $(15 \pm 6.2$ in vehicle-treated vs. $60.6 \pm 8.5$ in PTHtreated $P T H 1 R^{f / W T}$ mice and $24.6 \pm 8.1$ in vehicle-treated vs. $18.6 \pm$ 7.23 in PTH-treated PTH1R $R^{f / f l}$ mice) (Figure 5, A-F). We observed very similar results by using flow cytometry analysis. We observed a significant increase in the number of $\mathrm{tdTomato}^{+}$cells after teriparatide administration in Sox9-creERT2; R26RTomato; PTH1R ${ }^{f / W T}$ mice compared with controls $\left(1.8 \times 10^{-3} \pm 3.54 \times 10^{-4}\right.$ in vehicletreated mice vs. $7.8 \times 10^{-3} \pm 6.3 \times 10^{-4}$ in teriparatide-treated mice) compared with vehicle- and teriparatide-treated mice $\left(1 \times 10^{-3} \pm\right.$ $3.5 \times 10^{-4}$ in vehicle-treated mice vs. $2.2 \times 10^{-3} \pm 2.8 \times 10^{-4}$ in teriparatide-treated mice) in Sox9-creERT2; R26RTomato; PTH1R $R^{f / f l}$ mice (Figure 5, G-I). Thus, knocking out the PTH/PTHrP receptor from Sox9-creERT $2^{+}$mesenchymal precursors led to abrogation of the effects of teriparatide administration, suggesting that teriparatide acts directly on Sox9-creERT2 $2^{+}$mesenchymal precursors in vivo.

Withdrawal of intermittent teriparatide administration leads to adipocytic differentiation of Sox9-creERT2+ multipotent cells. Bones lose substantial amounts of their mass and cellular constituents when intermittent teriparatide regimens are halted (10), but there has been little analysis of cell fates in that setting. To determine the fates of cells descended from Sox9-creERT2-expressing cells after stopping teriparatide administration, we designed a protocol in which Sox9-creERT2; R26RTomato, Ocn-GFPtpz mice received a single 2-mg tamoxifen injection at 6 to 7 weeks of age and, starting 24 hours later, these mice were subjected to daily teriparatide administration for 8 weeks. A second group of mice received intermittent teriparatide administration for only 4 weeks and was given vehicle for 4 more weeks. A third group was given vehicle for 8 weeks (Figure 6A). Sections from tibia of mice that received intermittent PTH 1-34 administration for 4 weeks showed substantial trabecular bone formation (Figure 6B). Similarly, teriparatide-treated mice at 8 weeks showed robust bone deposition (Figure $6 \mathrm{C}$ ). Vehicle-treated mice, as expected, showed no changes (Figure 6D). Mice that received teriparatide for 4 weeks and then vehicle for 4 weeks showed no obvious morphological differences in trabecular or cortical bone from those treated with vehicle only (Figure 6E). Interestingly, we observed that the metaphyseal marrow and diaphysis appeared to contain large numbers of adipocytes (Figure 6, $\mathrm{E}$ and $\mathrm{H}$ ) not seen in either the mice given teriparatide for 8 weeks or those given only vehicle (Figure 6, C, $\mathrm{D}$, and $\mathrm{F}$ ); this finding was confirmed by oil red $\mathrm{O}$ staining (Figure $6, \mathrm{G}$ and I) and osmium tetroxide staining using $\mu \mathrm{CT}$ (Figure 6J). Lineage tracing showed a large number of tdTomato ${ }^{+}$cells in the primary and secondary spongiosa in mice treated with teriparatide for 8 weeks (Figure 6K). Vehicle-treated mice also showed several tdTomato $^{+}$cells that colocalized with GFPtpz protein and were present on bone surfaces (Figure 6L). However, mice that were withdrawn from teriparatide also showed metaphyseal adipocytes that descended from Sox9-creERT2 ${ }^{+}$cells (Figure 6M and insert).
Confocal analysis revealed a significant increase in the numbers of adipocytes in the metaphysis of mice from whom teriparatide had been withdrawn (148 \pm 28 vs. 0$)$; many of these adipocytes were tdTomato $^{+}(28 \% \pm 4 \%)$ (Figure 6O). These findings suggest that Sox9-creERT2 cells are capable of differentiating into adipocytes in vivo and are consistent with in vitro findings as well (Supplemental Figure 7). Thus, the increase seen in trabecular bone after several weeks of teriparatide (Figure 6B, for example) disappears within 4 weeks of teriparatide withdrawal; instead, adipocytes, some of which are descended from Sox 9 -creERT2 ${ }^{+}$cells generated 8 weeks earlier, accumulate in marrow.

The experimental design of the PTH withdrawal experiment, with a prolonged chase after pulsing Sox9-creERT2-expressing cells 8 weeks before sacrifice, led necessarily to TdTomato expression in osteoblast precursors, osteoblasts, and osteocytes. We wondered whether cells rather late in the lineage might contribute to the fate plasticity demonstrated by this experiment. Therefore, we performed withdrawal experiments (Supplemental Figure 9A) in mice that expressed an Ocn promoter-driven creER along with a TdTomato reporter to mark osteoblasts expressing osteocalcin, a marker of well-differentiated osteoblasts. We observed $\mathrm{Ocn}$-creER ${ }^{+}$cells as osteoblasts and osteocytes in both vehicle- and PTH-treated mice (Supplemental Figure 9, B and C). Interestingly, after withdrawal of teriparatide, $\mathrm{Ocn}$-creER $\mathrm{R}^{+}$adipocytes that costained with perilipin accumulated in the marrow (Supplemental Figure 9, D and E).

Because Wnt signaling has been documented as affecting the fates of cells in the osteoblast lineage (11) and PTH signaling increases activation of $\beta$-catenin, we further hypothesized that withdrawal of PTH may decrease $\beta$-catenin signaling in bone, especially in Sox9-creERT2 ${ }^{+}$cells, and thereby contributes to the switch in cell fate. Mice treated with teriparatide for 8 weeks showed a robust activation of $\beta$-catenin in Sox 9 -creERT2 ${ }^{+}$cells (Supplemental Figure 10C) as compared with vehicle-treated mice (Supplemental Figure 10B) after staining with an Ab specific for the active, nonphosphorylated $\beta$-catenin. Interestingly, mice that were withdrawn from teriparatide showed a dramatic suppression of $\beta$-catenin activity (Supplemental Figure 10D).

\section{Discussion}

Herein, we show that Sox9-creERT2 ${ }^{+}$cells in adult mice can give rise to osteoblasts, adipocytes, and $\mathrm{CXCL}^{2} 2^{+}$reticular stromal cells. We also show that Sox 9 -creERT2 ${ }^{+}$cells identified 2 days after tamoxifen administration are immature osteoprogenitor cells that do not express the early transcription factor, osterix, but differentiate into osterix-expressing cells after several days.

We have now used these cells to study the effects of teriparatide on osteoblast precursors, utilizing a genetic approach. By marking only immature mesenchymal cells in adult mice, we provide in vivo evidence that $\operatorname{Sox} 9$-creERT2 ${ }^{+}$cells are sources of mature osteoblasts that also take part in the teriparatide-mediated increase in osteoblast numbers and bone-forming activity when administered once daily. Our data suggest that the early precursors of the osteoblasts express PTH receptors that play a crucial role in the very early stages of the osteoblast lineage and that these receptors in the early precursors are required for the action of teriparatide to increase the numbers of osteoblast precursors 
A
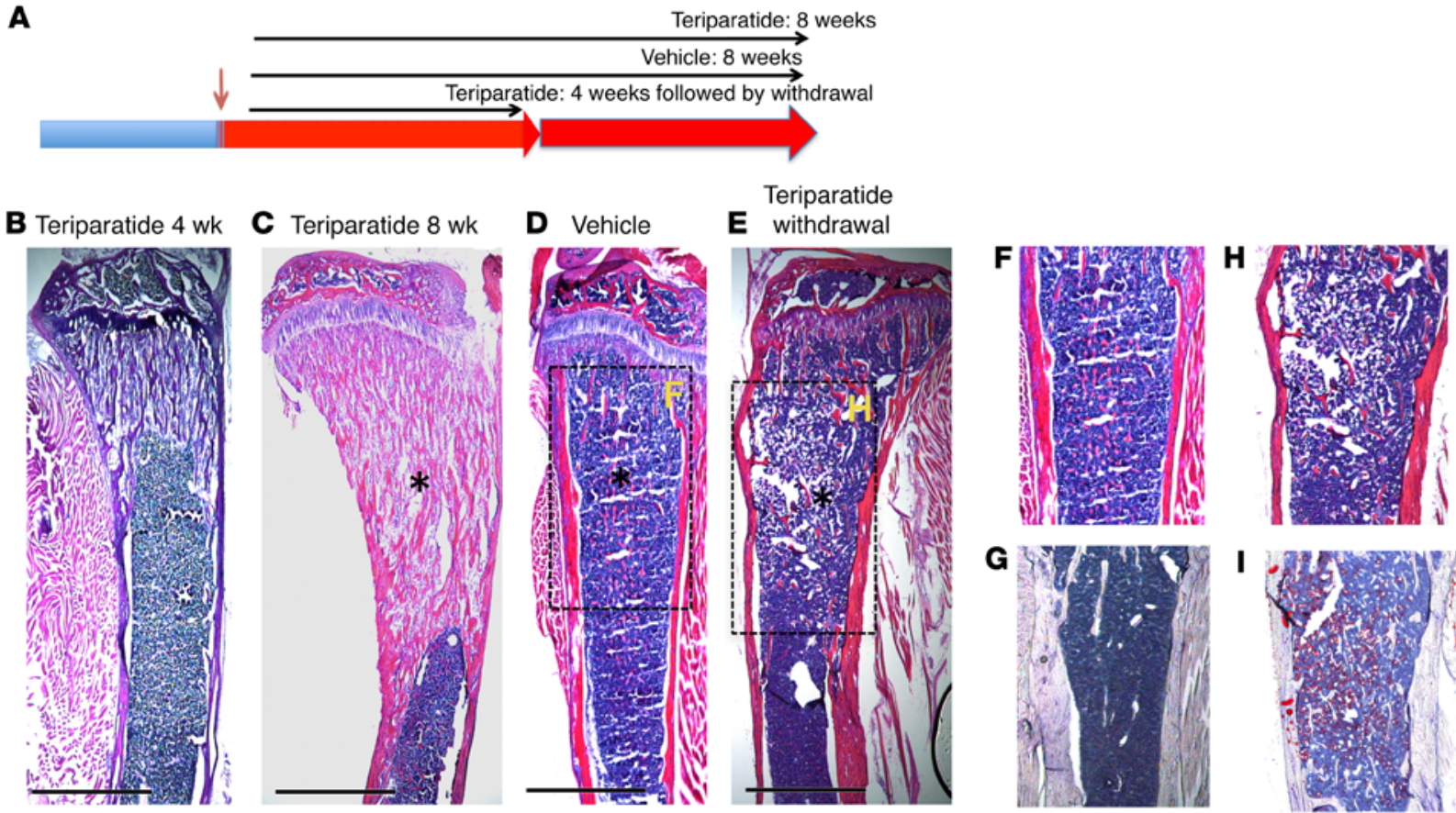

\section{$\mathbf{J}$}

Osmium tetroxide PTH-withdrawal
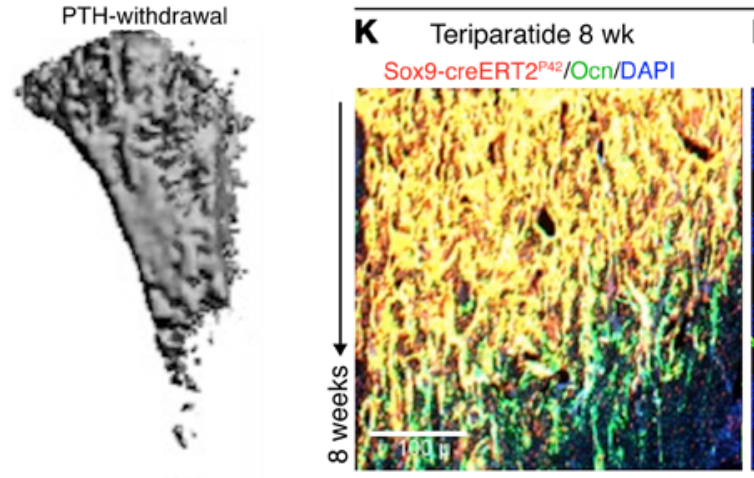

Sox9-creERT2; R26RTomato, Ocn-GFPtpz

L Vehicle Sox9-creERT2 $2^{\mathrm{p}+2} / \mathrm{Ocn} / \mathrm{DAPI}$

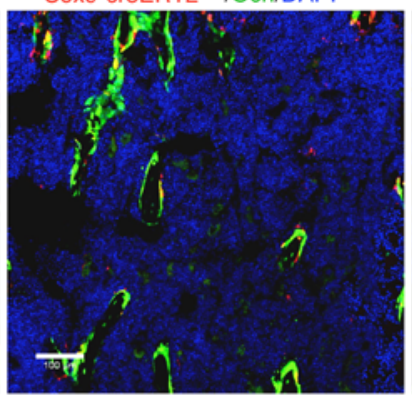

M Teriparatide withdrawal Sox9-creERT2 ${ }^{\mathrm{p}+2} / \mathrm{Ocn} / \mathrm{DAPI}$

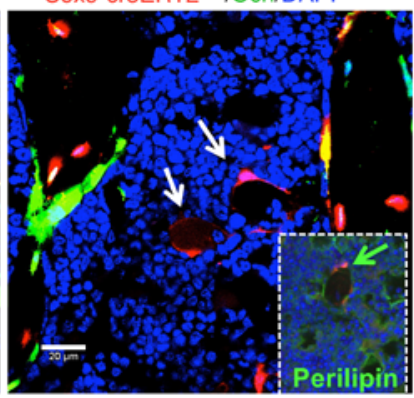

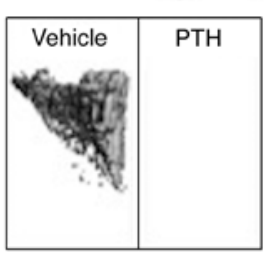
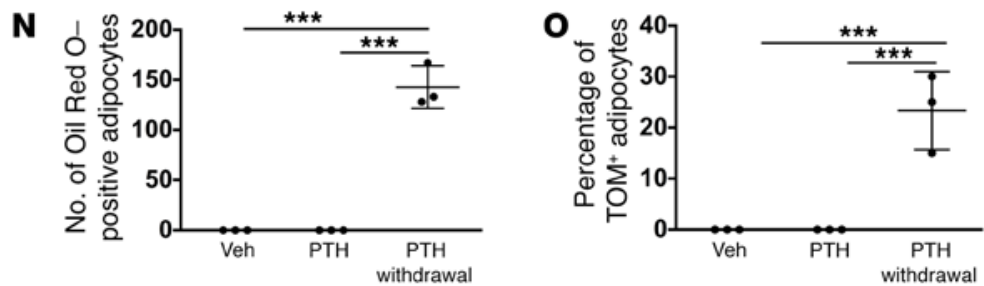

Figure 6. Withdrawal of teriparatide administration leads to adipocytic differentiation of Sox9-creERT2+ multipotential cells. (A) The protocol used in the experiment to study the fate of Sox9-creERT2+ cells after PTH 1-34 withdrawal. Mice received a single tamoxifen injection at P42. Twenty-four hours later, mice were subjected to either vehicle or PTH 1-34 once daily for 30 days. Mice were sacrificed and long bones were harvested for evaluation by histology, epifluorescence microscopy, and confocal microscopy. Each panel reflects data from 3 mice/genotype from 3 independent experiments. (B-E) Representative $H \& E$ sections of tibia from mice showing increased trabecular bone in mice that received teriparatide every day for 4 weeks (B) and 8 weeks (C), mice that received vehicle (D), and mice that were withdrawn from teriparatide administration after 4 weeks (E), but evaluated 4 weeks thereafter. $\mathbf{F}$ and $\mathbf{H}$ show magnified images of boxes in $\mathbf{D}$ and $\mathbf{E}$. Scale bars: $500 \mu \mathrm{m}$. Asterisks show trabecular bone differences in different mice. Oil red $\mathbf{O}$ staining of metaphysis from vehicle (G) and teriparatide withdrawal (I). (J) Osmium tetroxide staining of bone marrow fat in teriparatide-withdrawal, vehicle, and teriparatide-treated mice visualized using $\mu \mathrm{CT}$. (K-M) Representative section of tibia after lineage tracing of adult Sox9-creERT2 mice. Sox9-creERT2; R26RTomato mice showing cells in the trabecular bone of PTH 1-34-treated (K) and vehicle-treated mice (L) and mice that were withdrawn from teriparatide (M). Mice that were withdrawn from PTH 1-34 show TdTomato+ adipocytes (arrows). Insert in $\mathbf{M}$ shows coexpression of perilipin with Sox9-creERT2 ${ }^{+}$adipocyte after teriparatide withdrawal (green arrows). Each panel reflects data from 3 mice/genotype from 3 independent experiments. Scale bars: $100 \mu \mathrm{m}$ ( $\mathbf{K}$ and $\mathbf{L}) ; 20 \mu \mathrm{m}$ ( $\mathbf{M}$ and insert). ( $\mathbf{N}$ and $\mathbf{0}$ ) The number of oil red $\mathrm{O}$ adipocytes in the entire bone marrow $(\mathbf{N})$ and percentage of adipocytes that were descendants of Sox9-creERT2; R26RTomato cells (0). Data represent mean \pm SD from 3 independent experiments with 3 mice/experiment. ${ }^{* *} P<0.0001$. Statistical evaluation was done by nonparametric 2-tailed Student's $t$ tests, and the data were subjected to Bonferroni's correction for multiple testing. 
and their descendants. Somewhat surprisingly, the increase in the numbers of these precursors occurs in association with substantial suppression of apoptosis, without detectable effects of teriparatide on the rate of proliferation of the precursors. In this way, the action of teriparatide on osteoblast precursors resembles that of PTH on mature osteoblasts.

Previous studies have examined the action of PTH on putative osteoblast precursors. For example, Nishida et al. (12) show that intermittent PTH administration to rats increased the number of colonies grown in vitro after plating marrow stromal cells (CFU-F). In studies such as these, the effects of proliferation versus apoptosis on the colony-originating cells cannot be distinguished. Further, cells capable of forming CFU-F from marrow may be heterogeneous and are likely to be distinct from the Sox9-creERT2-marked cells studied here. Our previous work, for example, showed that collagen II-Cre; tdTomato marks precursors of cells that eventually form CFU-Fs in young postnatal mice, but that the collagen IIcreER transgene is no longer expressed in the CFU-F-originating cell when tamoxifen is given postnatally (8). More recently, Pettway et al. (13) showed that marrow stromal cells, when injected into mice with implanted bone "ossicles," increased their proliferation in response to administration of teriparatide; most of these stromal cells are likely to be more advanced in the early osteoblast lineage than the cells we analyzed early after tamoxifen administration. Méndez-Ferrer (6) showed that administration of teriparatide increases proliferation of cells marked with nestin-GFP, though those cells are clearly a heterogeneous population that overlaps somewhat with Sox9-creERT2 cells, but also includes endothelial cells as well (14). The heterogeneity of all these findings probably reflects the heterogeneity of assays used to detect cells at various stages of differentiation in marrow. Mature cells of the osteoblast lineage secrete growth factors such as IGF-1, Wnts, and fibroblast growth factors and show suppression of sclerostin gene expression in response to teriparatide; all of these agents represent potential indirect mediators of teriparatide action on Sox9-creERT2-marked cells. Our data show, however, that if these growth factors from mature osteoblasts/osteocytes do act on Sox9-creERT2-marked cells, the direct action of PTH on these cells is required for the actions of growth factors to be manifested.

Long-term PTH therapy has been shown to cause osteosarcoma in rats (15); however, there is currently no cellular explanation of such an effect of the hormone. Our findings of suppression in the rate of apoptosis in the early progenitor cells, if sustained over the long term, might contribute to the vulnerability of such cells to undergoing malignant transformation.

It has been known that PTH suppresses adipocytic differentiation of human stromal cells in vivo (16) and that PTHrP haploinsufficient animals show increased bone marrow adiposity in vivo (17). It is well established that bone mass falls after cessation of teriparatide treatment of osteoporotic patients (18), though nothing is known about possible adipocyte accumulation after cessation of teriparatide in humans. We provide evidence that sudden cessation of teriparatide after prolonged administration may lead to adipocytic differentiation; the physiologic consequences of this fate switch are unknown. Here, we show that some of these adipocytes are descendants of Sox9-creERT2 cells. We also show that, after PTH withdrawal, some of the adipocytes can descend from Ocn-cre-expressing cells, suggesting striking plasticity even of mature osteoblasts. This conversion of Sox9-creERT2 or OcncreER cells to adipocytes does not occur when PTH administration continues, suggesting that the option of becoming an adipocyte is suppressed by PTH administration.

Our group has previously shown that Wnt/ $\beta$-catenin is a crucial fate determinant of osteoblast versus adipocytic lineage in Os $x$-creER ${ }^{+}$cells (11). Teriparatide administration, both in vitro and in vivo, increases Wnt/ $\beta$-catenin signaling. Therefore, we hypothesized that teriparatide withdrawal might lead to a sudden decrease in Wnt/ $\beta$-catenin signaling in Sox9-creERT2 ${ }^{+}$ cells in vivo. To assess the presence of active form of $\beta$-catenin, we stained Sox9-creERT $2^{+}$cells with an Ab that only detects the nonphosphorylated (active) form of $\beta$-catenin that has not been phosphorylated by GSK-3 and thus is functionally active in the canonical Wnt-signaling pathway. As predicted, we observed a dramatic increase of active $\beta$-catenin in Sox9-creERT2 ${ }^{+}$cells in teriparatide-treated mice when compared with the levels in mice after PTH withdrawal (Supplemental Figure 10). Our data indicate that Wnt/ $\beta$-catenin may thus be an important determinant of fate switch after teriparatide withdrawal.

Other signaling pathways may also be important in regulating the switch from osteoblast to adipocyte after PTH withdrawal. PTH suppresses PPAR $\gamma$, a crucial transcription factor that leads to adipocytic differentiation of MSCs in vitro and in vivo (16). PTH also regulates several zinc finger proteins, ZFP521 and ZFP423, which promote osteoblast differentiation at the expense of adipocyte differentiation (19). Further, the possibility that expansion of adipocytes occurs in people stopping teriparatide therapy should be explored.

\section{Methods}

Mice.Ocn-GFP/tpz (20), nestin-GFP (6), CXCL12-GFP (21), Osx-mCherry (JAX024850), Sox9-creERT2 (22), Rosa26-loxP-stop-loxP-tdTomato (JAX7914), Col2a1-creER (23), aggrecan-creERT2 (JAX019148), PTH receptor 1 flox/flox (24), Ocn-creERT (25), and Rosa26-loxP-stop-loxPZsgreen1 mice (JAX 007906) were used. Unless otherwise mentioned, in all of our experiments, 6- to 7-week-old mice received $2 \mathrm{mg}$ tamoxifen once intraperitoneally 24 hours before the subcutaneous administration of PTH 1-34. Tamoxifen was dissolved first in 100\% ethanol, then in sunflower seed oil (Sigma-Aldrich, S5007) overnight at $60^{\circ} \mathrm{C}$. To examine the effect of PTH, the mice were given daily subcutaneous injections of vehicle (10 $\mathrm{mM}$ citric acid, $150 \mathrm{nM} \mathrm{NaCl} / 0.05 \%$ Tween-80) or $400 \mathrm{ng} / \mathrm{g}$ body weight of PTH 1-34. Mice were genotyped by PCR. Animals were housed together in a temperature- and humidity-controlled animal vivarium and maintained under an ad libitum food and water diet supplied by the Center of Comparative Medicine of Massachusetts General Hospital.

Histology. Samples were dissected under a dissecting microscope (Nikon SMZ-10A) to remove soft tissues and fixed in $4 \%$ paraformaldehyde, kept overnight at $4^{\circ} \mathrm{C}$, then decalcified in 15\% EDTA for 7 to 14 days. Decalcified samples were cryoprotected in $30 \%$ sucrose/PBS followed by a $30 \%$ sucrose/PBS:OCT (1:1) solution, each kept overnight at $4^{\circ} \mathrm{C}$, embedded in OCT, and cryosectioned at $15 \mu \mathrm{m}$ (Leica CM1850). Images were captured with an epifluorescence microscope (Nikon Eclipse E800) with prefigured triple-band filter settings for DAPI/FITC/TRITC and merged with Spot Advanced Software 
(Spot Imaging) or with an automated fluorescent microscope with a whole-slide scanning platform (TissueFAXS, TissueGnostics). Confocal images were acquired using LSM510 and Zen2009 software (Zeiss) with lasers and corresponding band-pass filters for DAPI (Ex.405nm, BP420-480), GFP (Ex.488nm, BP505-530), and tdTomato (Ex.543nm, BP565-595). An LSM Image Viewer was used to capture and align images. Representative images of at least 3 independent biological samples are shown in the figures. Routine H\&E and oil red O staining were performed using previously published protocols (11).

Flow cytometry. Femurs were carefully dissected and crushed in 2 $\mathrm{ml} \mathrm{HBSS}$, and supernatants were filtered into the 50-ml Falcon tubes. Tissue remnants were incubated with 2 Wunsch units of Liberase TM (Roche) at $37^{\circ} \mathrm{C}$ for 30 minutes on a shaking incubator (Thermomixer, Eppendorf). 0.25\% Trypsin-EDTA (Gibco, Thermo Fisher Scientific) was added to harvest osteoblasts. Cells were mechanically triturated using an 18-gauge needle and a 1-ml syringe (BD) and filtered into 1 tube. Cells were stained with anti-mouse CD45-APC and DAPI in Dulbecco's PBS (DPBS) $/ 2 \%$ FBS on ice for 30 minutes. Flow cytometry was performed using a 4-laser BD LSRII (Ex.355/407/488/633nm) flow cytometer and FACSDiva and analyzed on FlowJo (TreeStar). Representative plots of at least 3 independent biological samples are shown in the figures. The percentages have been calculated as previously described (7).

Immunohistochemistry. Cryosections were postfixed in $4 \%$ paraformaldehyde for 15 minutes, blocked with 2\% BSA for 30 minutes, and incubated with rat anti-Sox9 monoclonal Ab (1:100) (AB5535, Millipore), non-phospho- $\beta$-catenin (active) (Ser33/37/Thr41) (D13A1), rabbit mAb 8814 (1:200, Cell Signaling Technology), anti-perilipin Ab (1:500, Santa Cruz Biotechnology Inc.) (sc-67174), FABP4 (1:500, Abcam) (ab 92501), and adiponectin Ab (1:200, provided by Clifford Rosen, Maine Medical Center Research Institute, Scarborough, Maine, USA), non-phospho- $\beta$-catenin rabbit mAb (8814) overnight at $4^{\circ} \mathrm{C}$, and subsequently, with Alexa Fluor 488 -conjugated goat anti-rat $\operatorname{IgG}$ (Invitrogen A21087) for 3 hours at $4^{\circ} \mathrm{C}$. Sections were further incubated with DAPI to stain nuclei and observed under the microscope.

Cell proliferation and apoptosis assays. To evaluate cell proliferation, 1.5 mg 5-ethynyl-2'-deoxyuridine (EdU) (Invitrogen A10044) dissolved in PBS was administered to adult mice 6 hours before sacrifice. Cells were isolated as described in the flow cytometry section. The Click-iT Imaging Kit (Invitrogen, C10337) with Alexa Flour 488azide (Invitrogen, A10277) was used to detect EdU-positive cells by flow cytometry. Apoptotic cells were detected using a commercially available kit that identifies cells surface annexin $\mathrm{V}$ using flow cytometry (BioLegend, 640922).

Quantitative reverse-transcription-PCR. To determine the levels of transcripts encoding Sox 9 and PTH1R, tdTomato-positive cells were isolated after manually removing the growth plate under a dissection microscope to avoid chondrocytic contamination and sorted using a
BD FACSAria sorter for tdTomato ${ }^{+}$and Ocn-GFPtpz cells. From 200 to 300 cells were FACS sorted per tube, and RNA was isolated, followed by amplification, reverse-transcription, and quantitative reverse-transcription-PCR (RT-PCR) per the manufacturer's instructions using a commercially available kit (Single Cell-to-CT qRT-PCR Kit, Ambion, 4458236). PCR was performed with primers from Assays-on-Demand (Applied Biosystems) on an ABI 7500 Prism system. The transcript levels were normalized to those for GAPDH. For quantitative PCR, the following primers from Assays-on-Demand were used: Sox9 (Mm00448840_ m1), PTH1R (Mm00441046_m1), and Gapdh (Mm99999915_g1).

Osmium tetroxide staining. To assess marrow adipose tissue, the tibias of PTH-depleted mice were infiltrated with osmium tetroxide and evaluated by $\mu$ CT (SCANCO Medical AG) as described (26).

See Supplemental Methods for additional information.

Statistics. Results were represented as mean \pm SD. Statistical evaluation was done by nonparametric 2-tailed Student's $t$ test using GraphPad Prism version 6 for Mac (www.graphpad.com). Bonferroni's correction for multiple testing was used for Figure 2D, Figure 5, I and J, and Figure 6, N and O. $P<0.05$ was considered significant.

Study approval. All animal experiments were approved by the Institutional Animal Care and Use Committee of Massachusetts General Hospital.

\section{Author contributions}

DHB contributed by designing, performing experiments, acquiring and analyzing data, and writing the manuscript. NO contributed by acquiring and analyzing data and writing the manuscript. HMK contributed by designing, acquiring and analyzing data, and writing the manuscript.

\section{Acknowledgments}

We thank the MGH-Harvard Stem Cell Institute Flow Core Facility and Thomas Diefenbach of the Ragon Institute Confocal Core Facility. The authors are thankful to Sophia Trinh for her technical support and mouse line maintenance and the Endocrine Unit of Massachusetts General Hospital. We also thank Hang Lee, biostatistician, Massachusetts General Hospital, for verifying the statistical analysis used in the experiments. The work is funded with the help of NIH grants DK011794, AR066261, and P30 AR066261 (to HMK), an Early Investigator fellowship grant by Swiss National Science Foundation, and the Pfizer Aspire Young Investigator Grant (to DHB).

Address correspondence to: Henry M. Kronenberg, Endocrine Unit, Massachusetts General Hospital and Harvard Medical School, 50 Blossom Street, Boston, Massachusetts 02114, USA. Phone: 617.726.3967; Email: hkronenberg@mgh.harvard.edu.
1. Heaney RP. Vitamin D and parathyroid hormone. In: Bilezikian JP, Marcus R, Levine MA, Marcocci C, Silverberg SJ, Potts JT, eds. The Parathyroids: Basic and Clinical Concepts. Cambridge, MA: Academic Press; 2015:633-640.

2. Jilka RL. Molecular and cellular mechanisms of the anabolic effect of intermittent PTH. Bone. 2007;40(6):1434-1446.

3. Jilka RL, Weinstein RS, Bellido T, Roberson P, Parfitt AM, Manolagas SC. Increased bone formation by prevention of osteoblast apoptosis with parathyroid hormone. JClin Invest. 1999;104(4):439-446.

4. Kim SW, et al. Intermittent parathyroid hormone administration converts quiescent lining cells to active osteoblasts. J Bone Miner Res. 2012;27(10):2075-2084.

5. Bianco P, Robey PG. Skeletal stem cells. Development. 2015;142(6):1023-1027.

6. Méndez-Ferrer S, et al. Mesenchymal and hae- matopoietic stem cells form a unique bone marrow niche. Nature. 2010;466(7308):829-834.

7. Ono N, Kronenberg HM. Bone repair and stem cells. Curr Opin Genet Dev. 2016;40:103-107.

8. Ono N, Ono W, Nagasawa T, Kronenberg HM. A subset of chondrogenic cells provides early mesenchymal progenitors in growing bones. Nat Cell Biol. 2014;16(12):1157-1167.

9. Sugiyama T, Kohara H, Noda M, Nagasawa T. Maintenance of the hematopoietic stem cell 
pool by CXCL12-CXCR4 chemokine signaling in bone marrow stromal cell niches. Immunity. 2006;25(6):977-988.

10. Cohen A, et al. Bone density after teriparatide discontinuation in premenopausal idiopathic osteoporosis. JClin Endocrinol Metab. 2015;100(11):4208-4214.

11. Song L, Liu M, Ono N, Bringhurst FR, Kronenberg HM, Guo J. Loss of wnt/ $\beta$-catenin signaling causes cell fate shift of preosteoblasts from osteoblasts to adipocytes. J Bone Miner Res. 2012;27(11):2344-2358.

12. Nishida $\mathrm{S}$, et al. Increased bone formation by intermittent parathyroid hormone administration is due to the stimulation of proliferation and differentiation of osteoprogenitor cells in bone marrow. Bone. 1994;15(6):717-723.

13. Pettway GJ, et al. Anabolic actions of PTH (1-34): use of a novel tissue engineering model to investigate temporal effects on bone. Bone. 2005;36(6):959-970.

14. Mizoguchi T, et al. Osterix marks distinct waves of primitive and definitive stromal progenitors during bone marrow development. Dev Cell. 2014;29(3):340-349.
15. Watanabe A, et al. Osteosarcoma in SpragueDawley rats after long-term treatment with teriparatide (human parathyroid hormone (1-34)). J Toxicol Sci. 2012;37(3):617-629.

16. Rickard DJ, et al. Intermittent treatment with parathyroid hormone (PTH) as well as a nonpeptide small molecule agonist of the PTH1 receptor inhibits adipocyte differentiation in human bone marrow stromal cells. Bone. 2006;39(6):1361-1372.

17. Amizuka N, et al. Haploinsufficiency of parathyroid hormone-related peptide (PTHrP) results in abnormal postnatal bone development. Dev Biol. 1996;175(1):166-176.

18. Stroup J, Kane MP, Abu-Baker AM. Teriparatide in the treatment of osteoporosis. Am J Health Syst Pharm. 2008;65(6):532-539.

19. Kang S, et al. Regulation of early adipose commitment by Zfp521. PLoS Biol. 2012;10(11):e1001433.

20. Bilic-Curcic I, et al. Visualizing levels of osteoblast differentiation by a two-color promoterGFP strategy: Type I collagen-GFPcyan and osteocalcin-GFPtpz. Genesis. 2005;43(2):87-98. 21. Ara T, Tokoyoda K, Sugiyama T, Egawa T, Kawa- bata K, Nagasawa T. Long-term hematopoietic stem cells require stromal cell-derived factor-1 for colonizing bone marrow during ontogeny. Immunity. 2003;19(2):257-267.

22. Soeda T, Deng JM, de Crombrugghe B, Behringer RR, Nakamura T, Akiyama H. Sox9-expressing precursors are the cellular origin of the cruciate ligament of the knee joint and the limb tendons. Genesis. 2010;48(11):635-644.

23. Nakamura E, Nguyen MT, Mackem S. Kinetics of tamoxifen-regulated Cre activity in mice using a cartilage-specific CreER(T) to assay temporal activity windows along the proximodistal limb skeleton. Dev Dyn. 2006;235(9):2603-2612.

24. Kobayashi T, et al. PTHrP and Indian hedgehog control differentiation of growth plate chondrocytes at multiple steps. Development. 2002;129(12):2977-2986.

25. Kim SW, et al. Sclerostin antibody administration converts bone lining cells into active osteoblasts. JBone Miner Res. 2017;32(5):892-901.

26. Scheller EL, et al. Use of osmium tetroxide staining with microcomputerized tomography to visualize and quantify bone marrow adipose tissue in vivo. Meth Enzymol. 2014;537:123-139. 